\title{
Module de formation basé sur l'approche par les tâches pour développer la compétence de la gestion de classe du FLE
}

\author{
Dr. Manal Mounir
}

Maître de conférences de Curricula et de Méthodologie du FLE

\section{Sommaire}

Le développement des compétences professionnelles lors de la formation initiale, n'est pas seulement une question d'appropriation de savoirs. Il faut que les capacités opérationnelles croissent et permettre aux futurs enseignants de maîtriser de mieux en mieux le champ pédagogique. C'est dans cette perspective que nous avons souhaité étudier la construction de la compétence de gestion de classe, une composante qui caractérise l'acte d'enseigner et qui se détermine par un ensemble d'actions destinées à établir un climat favorable aux apprentissages des élèves. Ce préalable ou ce complément à l'approche didactique des contenus consiste à instaurer des règles et mettre en place des dispositifs pour créer, maintenir et restaurer un environnement favorable à l'apprentissage.

Tout en considérant le caractère dynamique du développement d'une compétence, nous avons posé l'hypothèse que la construction de la compétence de gestion de classe s'initialise et se développe déjà pendant la formation initiale. Pour caractériser cette hypothèse, nous avons anticipé plusieurs éléments de réponse.

Mots-clés : module de formation, gestion de classe, approche par les tâches, enseignant du FLE 


\section{Introduction}

La gestion de classe est devenue une compétence reconnue de l'enseignant qui œuvre tant en formation initiale qu'en formation continue.

Le concept de gestion de classe s'est grandement transformé au cours des trois dernières décennies dans les recherches. Sa signification, réservée au départ à l'ordre et à la discipline dans la classe (McQueen, 1992; Burden, 1995), s'est peu à peu élargie pour englober maintenant l'ensemble des actes réfléchis, séquentiels et simultanés qu'effectuent les enseignants pour établir et maintenir un bon climat de travail et un environnement favorables à l'apprentissage (Archambault et Chouinard, 2003; Bowen, Desbiens, Gendron et Bélanger, 2005).

Le concept de gestion de classe renvoie désormais à tout ce qui préside à la planification et à l'organisation des situations d'enseignementapprentissage.

Des recherches empiriques sur cette question ont fait apparaître d'autres éléments cruciaux. Elles ont permis d'identifier des pertes du temps réel d'apprentissage liées à des répétitions de comportements et de consignes au début et à la fin d'une leçon et lors des transitions dans le déroulement d'une leçon (Kounin, 1970; Yinger, 1977; Arlin, 1979; Leinhardt, Weidman et Hammond, 1987; Jones, 1987; Brophy, 1988; Charlier, 1989; Struyk, 1991 ; Burden, 1995; Yorke, 1998).

La gestion de la classe, rarement appliquée, constitue une référence majeure pour les enseignants francophones. Elle est à l'arrière-plan de la pensée enseignante, comme une sorte d'idéal ou de mauvaise conscience.

L'étendue du concept de gestion de classe amène à la considérer comme une des compétences majeures nécessaires en début de carrière, une

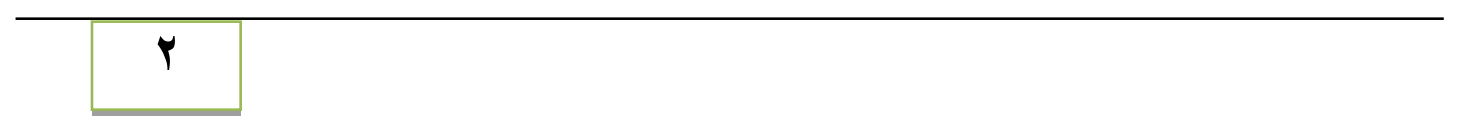


compétence indispensable à l'établissement de la confiance en soi de l'enseignant et de son identité professionnelle.

L'importance de la gestion de classe, surtout pour les enseignants débutants, s'explique par le fait que si cette compétence n'est pas maitrisée, il leur sera difficile d'acquérir les autres compétences nécessaires pour enseigner. Un enseignant débutant qui, en début d'année, ne réussit pas à instaurer ni à maintenir des routines relatives aux déplacements en classe et au bavardage, à orienter les élèves dans les étapes du déroulement d'une leçon ou à communiquer clairement les consignes pour accomplir une tâche, fera perdre beaucoup de temps et d'énergie à ses élèves. Ces nuisances à l'apprentissage découragent les élèves. De son côté, l'enseignant ne comprend pas pourquoi ses élèves n'apprennent pas. Son insatisfaction l'empêche de planifier des situations d'enseignement motivantes pour les élèves.

On pourrait évoquer bien d'autres exemples pour illustrer qu'un enseignant débutant qui ne maîtrise pas la compétence à gérer sa classe aura aussi des difficultés dans les autres facettes de son rôle, telles que celles de motivateur, de facilitateur, de négociateur ou d'organisateur.

En Egypte, malheureusement, la position traditionnelle typique consiste à dire que la compétence à gérer une classe s'acquiert par l'expérience, c'està-dire par essais et erreurs ou par imitation. Cette position mène alors à ne lui accorder pas une place dans les programmes de formation. Traditionnellement aussi, il s'agit d'une compétence purement personnelle; l'inclure dans un programme de formation serait peine perdue.

Dans les deux cas, la conséquence est la même: elle aboutit à négliger cet aspect dans la formation.

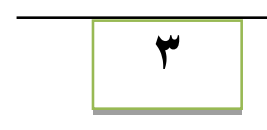


En ce qui concerne l'acquisition de cette compétence, les résultats de plusieurs recherches, comme (Emmer et Stough, 2001 ; Murdock et Miller, 2003), fondés sur une population d'enseignants ayant des niveaux d'expérience très variés, montrent que la gestion de classe fait problème à tous et pas seulement aux débutants.

La chercheuse a effectué une enquête consistant en un suivi d'un échantillon de 37 enseignants et enseignantes de secondaire, ceux-ci indiquent quels sont les objectifs les plus difficiles à atteindre pour eux. Une des conclusions est que «Faire travailler des élèves de niveaux hétérogènes et intéresser les moins motivés restent des difficultés, que n'ont pas toujours surmontées les enseignants ». Les plus grands besoins de ces enseignants sont les connaissances sur les façons de gérer les classes. De manière générale, on peut donc penser que, relativement à la plupart de ses aspects, la compétence de la gestion de classe ne découle pas purement et simplement de l'expérience. Malgré les efforts accomplis pour mettre en place une formation à l'acquisition de la compétence à gérer une classe, il n'en demeure pas moins qu'au long d'une carrière, un enseignant, même d'expérience, doit continuellement rajuster ses acquis en fonction de chaque nouvelle situation d'enseignement-apprentissage.

La question qui se pose alors est de savoir comment procéder pour aider les enseignants à développer leur compétence en ce domaine.

Ceci pose la question d'établir comment s'acquiert cette compétence.

La perspective actionnelle est une nouvelle perspective pour apprendre et enseigner les langues vivantes. Cette perspective constitue l'un des points clés de l'enseignement et de l'apprentissage des langues vivantes étrangères. 
L'enseignant doit considérer l'apprenant comme acteur de ses apprentissages. Il le met en activité et introduit à cette occasion la langue et la culture comme instruments d'action et non seulement de communication. On ne communique plus seulement pour parler avec l'autre comme le proposait l'approche communicative des années 1980 mais on communique pour agir avec l'autre.

Cette perspective représente un tournant dans l'enseignement et l'apprentissage des langues en ce sens qu'elle redéfinit le statut de l'apprenant et des tâches qu'il a à accomplir.

L'apprenant apprendra par la réalisation de tâches, mais il est important de garder à l'esprit qu'il n'y a tâche que lorsque l'apprenant est motivé par l'action proposée. De plus, la tâche finale, qui constitue l'aboutissement d'une séquence pédagogique, pourra être réalisée si l'apprenant est entraîné tout au long de la séquence au moyen d'activités lui permettant d'acquérir les outils dont il aura besoin. En travaillant à la réalisation d'une tâche, la langue sera utilisée comme outil de communication et conférera ainsi une dimension authentique à la situation d'apprentissage.

Le moyen d'action est fondé sur la motivation des apprenants, suscité par l'aboutissement d'une réalisation concrète (dite tâche finale ou complexe). Elle induit un ensemble de tâches intermédiaires, dans lesquelles tous les apprenants peuvent s'impliquer et jouer un rôle actif, qui peut varier en fonction de leurs besoins et intérêts. Il s'agit de donner du sens à l'apprentissage en mobilisant leur intérêt en les impliquant dans la résolution d'un problème qui fait naître le besoin de connaissances et donc le désir d'apprendre. La mise en œuvre d'un projet permet d'atteindre des objectifs d'apprentissage identifiables figurant au programme. Elle permet

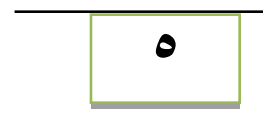


de développer des compétences (connaissances, aptitudes et habiletés) liés à la gestion du projet, ainsi que la socialisation des apprenants (apprentissage coopératif).

\section{Problématique de la recherche}

Le grand besoin des enseignants est l'acquisition des connaissances sur les façons de gérer leurs classes.

La recherche actuelle est censée répondre aux questions suivantes :

1. Quelles sont les composantes de la compétence de gestion de classe nécessaires pour les enseignants de FLE ?

2. Quel est le module de formation proposé à la lueur de l'approche par les tâches pour développer cette compétence de gestion de classe ?

3. Quel est le degré d'efficacité de ce module de formation ?

\section{Hypothèse de la recherche}

Il existe une différence significative entre la moyenne des notes de l'échantillon dans le pré/post test en faveur du post test en ce qui concerne la compétence de gestion de classe

\section{Objectif et Importance de la recherche}

Présenter la manière de structurer ce nouveau savoir, la gestion en classe, pour le transmettre ou le faire acquérir à l'étudiant-maître et en même temps ajuster le savoir à ses priorités et à ses exigences.

\section{Outils de la recherche}

1. Un module de formation proposé pour le développement de la compétence de gestion de classe

2. Un test pour mesurer le degré de développement de la compétence de gestion de classe

3. Une grille d'observation de la compétence de gestion de classe 


\section{Procédures de la recherche}

1. Etude de la compétence de gestion de classe

2. Etude de l'approche par les tâches

3. Elaboration des outils de la recherche

4. Application de l'expérimentation

5. Analyse et interprétation des résultats

\section{Terminologies de la recherche}

La gestion de classe : "Ensemble des pratiques éducatives auxquelles l'enseignant a recours afin d'établir, de maintenir et, au besoin, de rétablir dans la classe des conditions propices au développement des compétences des apprenants. » Archambault et Chouinard (2003)

\section{Définition opérationnelle}

La gestion de classe est un ensemble des actions qu'un enseignant conçoit, organise et réalise pour et avec ses apprenants (futurs-enseignants du FLE) afin de les engager, de les soutenir, de les guider et de les faire progresser dans leur apprentissage et leur développement dans leur formation initiale.

L'approche par les tâches : «L'approche par les tâches est de type actionnel en ce qu'elle considère avant tout l'apprenant comme des acteurs sociaux ayant à accomplir des tâches (qui ne sont pas seulement langagières) dans des circonstances et un environnement donnés, à l'intérieur d'un domaine d'action particulier. » Le Cadre européen commun de référence (CECR)

\section{Définition opérationnelle}

L'approche par les tâches est une conception où l'apprenant est un utilisateur qui accomplit des tâches : la classe est considérée comme le 
premier contexte situationnel dans lequel l'apprenant aura des tâches à accomplir pour acquérir la compétence de la gestion de classe.

\section{La gestion de classe}

\section{Introduction}

En enseignement, la gestion de classe est un élément incontournable pour assurer un climat de classe propice à l'apprentissage. On remarque d'ailleurs chez du nouveau personnel enseignant des inconforts reliés à la gestion de classe. Par contre, les enseignants d'expérience peuvent aussi vivre des événements pouvant ébranler leur gestion de classe pourtant bien établie.

Soulignons que les difficultés reliées à la gestion de classe est la principale cause d'abandon de la profession enseignante en début de carrière et qu'un mauvais climat de classe peut avoir un impact négatif sur la persévérance scolaire des apprenants.

La gestion de classe représente un défi pour plusieurs enseignants car elle est composée de plusieurs pratiques complexes qui doivent être actualisées souvent simultanément par l'enseignant. Tous n'ont pas la même approche. Ainsi, les enseignants se distinguent par leur profil personnel de gestion de classe, grandement influencé par leurs conceptions personnelles de l'enseignement et leurs représentations des besoins des élèves. Leur expérience, leur sexe, l'âge et le type d'élèves jouent également un grand rôle dans l'établissement de leurs différentes pratiques de gestion de classe. Plusieurs raisons ont motivé les chercheurs et les praticiens à étudier la gestion de classe. Deux motifs majeurs de s'intéresser à ce sujet. D'abord, la gestion de classe fait partie du quotidien de milliers d'enseignants et d'élèves, et un temps considérable est absorbé par des tâches de gestion. 
Ensuite, les conclusions des nombreuses recherches indiquent l'existence de relations significatives entre la gestion de classe et une variété de retombées telles que la réussite, les attitudes et le comportement des élèves ainsi que la satisfaction au travail et l'efficacité des enseignants.

Plusieurs chercheurs sont venus corroborer l'importance de la gestion de classe sur la réussite éducative et scolaire (Wang, Haertel et Walberg, 1994) mais, comme le font remarquer Levin et Nolan (2000).

Une gestion de classe efficace est inséparable d'un enseignement efficace.

\section{Les composantes de la gestion de classe}

Un modèle théorique a été développé pour tenter d'une part de nommer les composantes de la gestion de classe pour ensuite en faire une meilleure analyse. Ce modèle se compose de neuf éléments.

\section{Gestion de classe: modèle théorique}

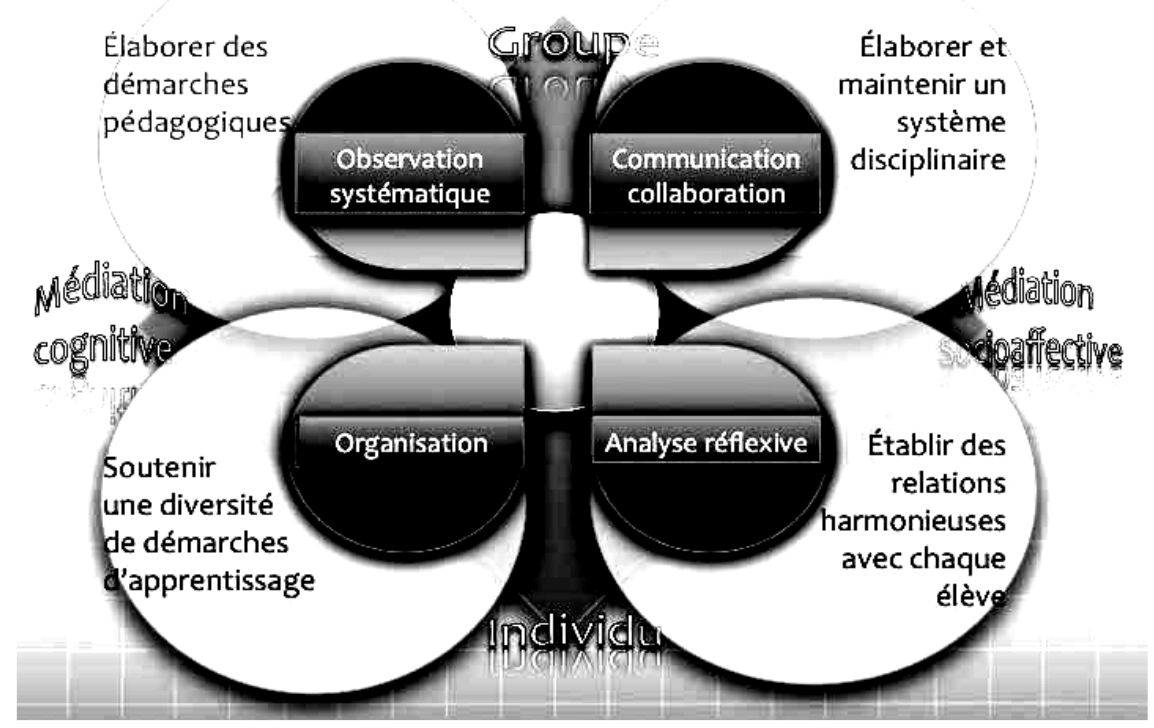

Figure 1 : Modèle théorique de gestion de classe (adapté par Lessard et Schmidt, 2008) 
$\mathrm{Au}$ cœur du modèle se retrouvent les valeurs. Ces valeurs influencent toutes les actions et les décisions de l'enseignant, notamment sur le plan des quatre éléments qui lui permettent de structurer son enseignement, soit l'organisation, l'observation systématique, la communication et collaboration et finalement, l'analyse réflexive.

L'enseignement consiste à l'élaboration de démarches pédagogiques, mais un enseignant efficace se préoccupe aussi de soutenir une diversité de démarches d'apprentissage chez les individus apprenants et de voir à ce que les besoins des élèves sur le plan socio-affectif soient comblés en établissant une relation harmonieuse avec chaque élève et en maintenant un système disciplinaire cohérent qui permet d'établir un climat propice à l'apprentissage.

\section{Le cœur du modèle de gestion : les valeurs}

Les valeurs des enseignants guident l'ensemble de leurs décisions face à la gestion de classe et teintent toutes les dimensions de leur pratique et ont un lien direct avec les huit autres éléments du modèle théorique. Par leurs attitudes et comportements à l'égard des élèves, les enseignants laissent transparaître leurs valeurs associées non seulement à la réussite scolaire, mais aussi à l'effort et à la vie dans la société formée par les membres du groupe-classe. En tant que guide et modèle, l'enseignant peut transmettre ces mêmes valeurs à ses élèves et promouvoir différentes conduites et habiletés sociales qui en découlent afin de favoriser l'établissement de relations harmonieuses dans la classe (Richardson et Fallona, 2001). De plus, les valeurs, attitudes et comportements de l'enseignant peuvent être intégrés par les élèves qui désireront l'imiter (Archambault et Chouinard, 2003). 
Ainsi, les valeurs que les enseignants ont à communiquer à leurs élèves sont : la gentillesse, l'équité, l'entraide, la coopération de l'apprentissage, l'empathie, le respect, l'autonomie, l'effort et le dépassement de soi.

\section{L'organisation}

Une bonne gestion de classe passe par l'ordre qui règne dans la classe. Plusieurs stratégies peuvent contribuer à y établir une organisation souple et efficace. Celles qui reviennent le plus fréquemment dans les écrits et qui ont été observées dans les pratiques de Calypso sont le partage des attentes, la gestion des transitions et les routines. En ayant recours à ces stratégies, l'enseignant développe un environnement structuré, ce qui permet aux élèves de se concentrer sur les apprentissages (Archambault et Chouinard, 2005; Johnson, Rice, Edgington et Williams, 2005).

Par conséquent, l'enseignant doit prendre le temps de réfléchir sur sa pratique et ses croyances personnelles afin d'identifier les conditions essentielles pour assurer la mise et place et le maintien d'une bonne organisation et d'un climat propice à l'apprentissage.

\section{L'observation systématique}

Que ce soit lors des leçons magistrales, du travail individuel ou en équipes, les enseignants efficaces supervisent l'ensemble de leurs élèves de façon à s'adapter à leurs besoins. Ils évaluent la compréhension et s'assurent de garder les élèves attentifs et engagés. En étant constamment à l'écoute et attentif à tout ce qui se passe dans la classe, ils récoltent les informations nécessaires pour maintenir un climat propice à l'apprentissage (Martineau et al., 1999). 
L'observation systématique permet également à l'enseignant de mieux connaître ses élèves de façon à ce qu'il puisse prendre des décisions adaptées aux caractéristiques et aux besoins de chacun.

\section{La communication et la collaboration}

En laissant une place aux élèves pour qu'ils s'expriment et en leur faisant pratiquer les habiletés de base pour transmettre un message (parler au «je», regarder et nommer la personne à laquelle je parle), l'enseignant montre qu'il accorde une place importante à la communication (Richardson et Fallona, 2001). Brown (2005) conseille à l'enseignant d'adopter une stratégie de communication active montre aux élèves qu'il les écoute vraiment à travers des signes verbaux et non verbaux et qu'il est sensible à ce qu'ils vivent. L'enseignant laisse aux élèves le temps de s'exprimer, il ne répond que lorsqu'ils ont terminé.

Les élèves aiment sentir que leur enseignant leur fait confiance et qu'il les considère suffisamment pour leur indiquer de façon transparente ce qu'il pense, leur transmettre un message qui dépeint réellement la situation, qu'il soit sincère dans ses échanges avec eux.

\section{L'analyse réflexive}

Inviter les élèves à pousser plus loin leur raisonnement, à expliquer le processus qu'ils ont utilisé, leur compréhension les amène à développer leur pensée et à approfondir leurs connaissances. En étant sensibilisé aux conséquences de leurs actions, ils sont plus à même de faire des choix réfléchis et appropriés (Beyda et al., 2002; Richardson et Fallona, 2001).

Questionner pour favoriser la réflexion: chez les enseignants cette catégorie est très représentative. Elle porte parfois sur les interventions didactiques et parfois sur les comportements des élèves. 


\section{L'établissement de relations harmonieuses}

Pour fournir un enseignement individualisé et intervenir efficacement auprès des élèves, l'enseignant doit savoir ce qui caractérise chacun: ses forces, ses difficultés, ses qualités et ses intérêts (Bowen et al., 2005; Bru et al., 2002; Klem et Connell, 2004; Muller, 2001). Le fait d'établir une relation chaleureuse, caractérisée par l'implication émotionnelle, peu de dépendance et de conflits (Harme et Pianta, 2001) avec chacun des élèves favorise leur motivation, leur réussite et le développement d'un sentiment d'appartenance à l'école (Lapointe et Legault, 2004; Murdock et Miller, 2003).

Certaines stratégies qui permettent de développer de bonnes relations sont notamment d'adopter et de faire adopter aux élèves des attitudes positives, de fournir des occasions aux élèves de connaître le succès et de développer leurs compétences et de les encourager à consigner leur progrès.

\section{L'élaboration et le maintien d'un système disciplinaire}

Pour assurer un environnement propice à l'apprentissage, l'enseignant doit, dès la rentrée scolaire, expliquer aux élèves quelles sont ses attentes au regard de leur comportement et faire en sorte, tout au long de l'année, qu'ils répondent à ces attentes (Archambault et Chouinard, 2003; Rosenberg et Jackman, 2003).

Le système de règles et de procédures favorise le maintien de l'ordre et de l'organisation en classe et fait en sorte que les élèves peuvent se concentrer sur le développement de leurs compétences.

\section{L'élaboration de démarches pédagogiques}

Les situations d'enseignement-apprentissage constituent le cœur de la vie dans une classe. La préparation de celles-ci favorise leur bon déroulement, 
l'atteinte des objectifs de l'enseignant et l'engagement des élèves (Archambault et Chouinard, 2003; Bowen et al., 2005).

Pour favoriser l'engagement des élèves, l'enseignant doit les inciter à s'impliquer dans les tâches scolaires, à chercher les réponses à toutes les questions, même si ce ne sont pas eux qui y répondent et à jouer un rôle actif dans la construction de leurs apprentissages.

\section{Le soutien d'une diversité de démarches d'apprentissage}

Dans le but de favoriser la réussite pour tous, l'enseignant doit idéalement adapter son enseignement aux différents styles d'apprentissage des élèves. Il accompagne les élèves pour les faire progresser à leur rythme et les amener à développer au maximum les compétences visées par le programme de formation (Archambault et Chouinard, 2003).

En amenant les élèves à devenir autonomes, à prendre leurs propres décisions et en leur donnant du pouvoir dans la classe, l'enseignant leur permet de se réapproprier leur démarche d'apprentissage.

\section{Conclusion}

Démontrer comment un enseignant arrive à établir un contexte dans lequel chaque élève peut atteindre son potentiel sur le plan de l'apprentissage et de son développement socio-affectif est très complexe.

Le modèle théorique de gestion de classe présenté démontre qu'un équilibre doit être atteint entre plusieurs tâches et fonctions pour qu'un enseignant arrive à aider les élèves à développer leurs compétences. L'enseignant doit se soucier à la fois du groupe, mais aussi des individus composant se groupe et il doit se soucier de leur développement cognitif et socio-affectif. Il doit établir et maintenir un climat propice à l'apprentissage tout en élaborant des démarches pédagogiques qui répondent aux divers 
besoins des apprenants. Son organisation et le recours à l'observation systématique, à une bonne communication et collaboration et finalement à l'analyse réflexive peuvent contribuer à rendre ce rôle un peu plus facile à gérer.

C'est pourquoi nous voyons l'importance d'un module de formation comme un moyen de soutenir le travail du personnel enseignant et, par le fait même, la réussite des apprenants.

\section{L'approche par les tâches}

\section{Introduction}

Le Cadre européen commun de référence (CECR) adopte une perspective actionnelle qui vise à préparer les apprenants à être des acteurs sociaux à travers la réalisation de tâches réelles (effectives) ou proches de la vie réelle (virtuelle) en classe de langue étrangère. Cette nouvelle approche, qui commence petit à petit à remplacer les exercices et les activités traditionnels, offre de nouvelles perspectives à la formation des enseignants.

Il est important pour nous de retenir qu'il n'y a tâche que si l'action est motivée par un objectif ou un besoin, personnel ou suscité par la situation d'apprentissage, si les apprenants perçoivent clairement l'objectif poursuivi et si cette action donne lieu à un résultat identifiable. On pense bien entendu immédiatement à la place centrale donnée à la tâche dans tous les actes pédagogiques.

\section{La nature de l'approche par les tâches}

L'approche par les tâches est centrée sur la notion de tâche et elle « considère avant tout l'usager et l'apprenant comme des acteurs sociaux 
ayant à accomplir des tâches dans des circonstances et un environnement donné, à l'intérieur d'un domaine d'action particulier ». (CECR, p.15)

Dans le cadre de cette approche, l'apprenant est un individu qui accomplit des tâches contextualisées à l'image du cadre social, professionnel... dans lequel il est ou sera inséré.

C'est dans ce sens que le CECR (p.15) souligne que «si les actes de parole se réalisent dans des actions langagières, celles-ci s'inscrivent ellesmêmes à l'intérieur d'actions en contexte social qui seules leur donnent leur pleine signification ».

Dans cette perspective, les activités de classe sont donc étroitement liées aux activités sociales que les apprenants sont ou peuvent être appelés à accomplir en société. De ces faits, dans le domaine de la formation des enseignants, on dit, plus facilement que l'apprenant a des tâches à accomplir et non pas des exercices à faire.

En effet, l'enseignant doit tenter d'importer en classe des situations propres au contexte social des apprenants et veiller à ce que les tâches réalisées soient transposables en contexte réel.

Par suite, l'enseignant est censé maîtriser finement les contextes sur lesquels reposent les tâches proposées aux apprenants. Et, naturellement, les apprenants sont censés se débrouiller dans ces contextes pour réaliser de façon optimale les tâches proposées.

Il est difficile de comprendre l'essence de la perspective actionnelle si on ne s'attarde pas sur la notion de tâche telle qu'elle est définie par le CECR. En outre, on peut difficilement cerner les fondements de cette perspective si, à la lumière du CECR, on n'établit pas une différence entre tâche et exercice. 
D'après le CECR «Les tâches ou activités sont l'un des faits courants de la vie quotidienne dans les domaines personnel, public, éducationnel et professionnel. L'exécution d'une tâche par un individu suppose la mise en œuvre stratégique de compétences données, afin de mener à bien un ensemble d'actions finalisées dans un certain domaine avec un but défini et un produit particulier $»$.

D'après la définition de tâche proposée par le CECR, l'apprenant est appelé à actualiser de façon stratégique un certain nombre de compétences devant une situation où il agit de façon réfléchie pour atteindre un but déterminé.

Nous pouvons ainsi considérer que la tâche sort l'apprenant de la dimension métalinguistique imposée par les traditionnels exercices. La tâche montre qu'en classe de langue, les activités réalisées sont avant tout des activités fondées sur l'interaction.

Notons enfin que la formation des enseignants fondé sur l'accomplissement de tâches favorise de toute évidence l'émergence de ce que nous appelons un apprenant réfléchi, c'est-à-dire un apprenant qui réfléchit en action et sur son action, un apprenant qui développe des stratégies afin d'optimiser son développement professionnel.

Pécheur, lors de son intervention au $7^{\mathrm{e}}$ congrès Panhellénique et International des Professeurs de Français à Athènes le 24 octobre 2010, schématise l'approche par les tâches comme suit :

\begin{tabular}{|l|l|}
\multicolumn{1}{c|}{} & Apprenant-utilisateur : acteur social (au centre de l'apprentissage) \\
\hline Tâches & Acquisition de savoir-faire, savoir, savoir-être, savoir-apprendre. \\
\hline & Contextes et situations \\
\hline
\end{tabular}

Schéma 1 : L'approche par les tâches selon Pécheur (2010) 
L'intérêt de cette schématisation de l'approche est triple : passer de l'homme en tant qu'acteur réagissant à un acteur agissant ; l'interaction entre l'utilisateur et l'environnement est pensée comme tâche à accomplir ; ce schéma vaut autant pour l'utilisateur que pour l'apprenant.

En effet, l'apprenant est un utilisateur qui accomplit des tâches : la classe est considérée comme le premier milieu social ou le premier contexte situationnel dans lequel l'apprenant aura des tâches à accomplir.

\section{Qu'est-ce qu'une tâche?}

Le concept de tâche n'est pas nouveau dans la didactique des langues; c'est aux recherches et aux didacticiens anglo-saxons, comme Nunan (1989), Willis (1996) et Ellis (2003) que l'on doit l'approche fondée sur la réalisation des tâches Task-Based learning (TBL). Le noyau central de cette approche par les tâches est que les apprenants soient confrontés activement à la résolution d'un problème. Ensuite, cette notion a été largement reprise par le CECR.

Nunan définit la tâche de la manière suivante : « une tâche est un plan de travail en classe qui implique les apprenants dans la compréhension, la manipulation, la production ou l'interaction » (1989 : $10)$.

Ellis, quant à elle, propose la définition suivante :

Une tâche est un plan de travail qui exige des apprenants qu'ils traitent de manière pragmatique dans le but de réaliser un résultat qui peut être évalué selon que le contenu des propositions a été réalisé de façon correcte ou appropriée. (2003:16)

Le CECR définit la notion de tâche ainsi : 
Toute visée actionnelle que l'acteur se présente comme devant parvenir à un résultat donné en fonction d'un problème à résoudre, d'une obligation à remplir, d'un but qu'on s'est fixé [...] Si l'on pose que les diverses dimensions ci-dessus soulignées se trouvent en interrelation, on pose aussi que tout acte d'enseignement d'une langue est concerné, en quelque manière, par chacune de ces dimensions : stratégies, tâches, textes, compétences individuelles, compétence langagière à communiquer, activités et domaines. (2005:16)

Cette définition adoptée par le CECR est très proche de celle formulée par Ellis, car on y retrouve la notion de résultat.

Ellis (2003) identifie plusieurs dimensions, que l'on a adaptées à notre manière, pour définir une tâche :

- La portée de la tâche : elle permet de distinguer la fonction sémantique de l'exercice et pragmatique de la tâche.

- L'approche à partir de laquelle la tâche est considérée : elle permet de distinguer le canevas imaginé par l'enseignant du processus effectivement mis en place par les apprenants. Ceci relève de la distinction entre la tâche centrée sur le sens et celle centrée sur la forme.

- L'authenticité de la tâche : elle prône des tâches présentant deux types d'authenticité : Authenticité situationnelle, lorsque la tâche correspond à des actions de la vie réelle, et Authenticité interactionnelle, lorsque la tâche permet, aux apprenants la mise en œuvre de processus présents dans la vie réelle.

- Les processus cognitifs mis en place lors de la réalisation d'une tâche : la réalisation d'une tâche engage un certain nombre de processus 
cognitifs, comme par exemple la sélection, le raisonnement, la classification, etc.

- Le résultat de la tâche : il est nécessaire, ici, de distinguer entre le résultat et l'objectif de la tâche. Le résultat se réfère à ce que les apprenants sont arrivés à faire une fois la tâche terminée. Par contre, l'objectif se réfère aux propositions pédagogiques de la tâche.

Nous remarquons à travers ces définitions de la tâche qu'il est généralement convenu que les tâches doivent avoir des objectifs, réalisés par l'engagement des participants dans un comportement axé sur le but.

\section{Les tâches et leur rôle dans la formation des enseignants}

Selon Ellis, beaucoup de linguistes, de didacticiens et de spécialistes reconnaissent les avantages de l'introduction des tâches en classe de langues étrangères. Elles peuvent être introduites de deux manières (2003 : 27) :

- soit on les introduit dans les méthodes traditionnelles d'enseignement (Task-supported language teaching);

- soit on élabore l'ensemble du cursus autour des tâches (Task-based language teaching)

Il est à souligner que dans les deux cas, l'utilisation des tâches dans la classe rend l'enseignement/apprentissage d'une langue étrangère plus communicatif et interactif. En effet, les tâches développent chez les apprenants la capacité d'une utilisation de la langue dans de vraies situations liées au monde réel.

Dans une situation qui suppose l'utilisation de la langue étrangère en voie d'acquisition, les tâches peuvent avoir deux fonctions $(2006: 38)$ : 
- une fonction interactionnelle qui sert à établir et à maintenir le contact. Elle doit donc impliquer une communication la plus réelle possible entre les apprenants ;

- une fonction transactionnelle servant à échanger de l'information entre les interlocuteurs.

Dans cette perspective, l'introduction des tâches en classe est justifiée dans la mesure où elle permet de créer de l'interaction par laquelle passe l'enseignement/apprentissage d'une langue étrangère, les tâches développent ces deux habilités chez les apprenants à travers la mise en œuvre de situations réelles.

Pour certains auteurs comme Nunan, les tâches sont placées au centre de toute conception du processus d'enseignement et d'apprentissage de langues étrangères. Nunan $(1989: 11)$ représente le statut qu'il donne à la tâche de la manière suivante :

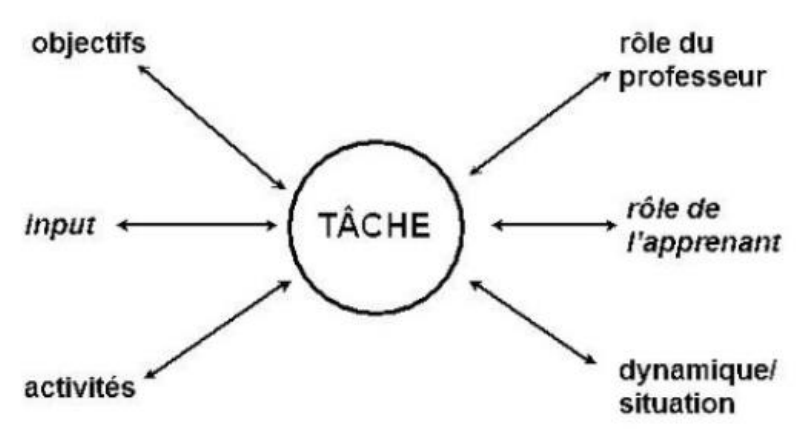

Schéma 2 : Le statut de la tâche selon Nunan (1989)

D'après ce schéma, nous remarquons que la conception d'une tâche exige la prise en considération de six paramètres : les objectifs, le support de départ (input), les activités que les apprenants doivent effectuer, les rôles respectifs de l'apprenant et de l'enseignant, et enfin la situation. 
D'autres auteurs, comme Willis et Ellis ajouteront un septième paramètre : le résultat, qui constitue pour les apprenants l'objectif de la tâche.

Le CECR considère l'approche par les tâches comme le centre de gravité de toute la conception du processus d'enseignement de langues étrangères. Cette approche permet de repenser l'enseignement en tenant compte des besoins des apprenants et en motivant le travail par une production finale.

Springer affirme que «la tâche pédagogique constitue l'élément moteur d'une rénovation pédagogique de type actionnel » (2009:33).

Le recours aux tâches en classe de langues étrangères doit s'appuyer aussi sur l'imaginaire et la créativité, ce qui facilite bien évidemment l'enseignement/l'apprentissage dans la mesure où les apprenants sont placés dans des situations proches du réel. Ces tâches simulées, bien qu'elles ne soient pas toujours identiques à la vie réelle, permettent aux apprenants de créer des interactions et des échanges.

\section{Le cycle de la tâche :}

La tâche s'offre à l'apprenant sous la forme d'un cycle de tâche : la prétâche, la tâche et la post-tâche.

Durant la pré-tâche, l'apprenant est amené à mobiliser les schémas mentaux et les scénarios lui permettant de planifier la tâche au niveau du contenu global, et de prévoir les éléments de la structure au niveau du scénario et du matériau à mobiliser ou à acquérir, nécessaire à l'exécution de la tâche.

Durant l'exécution de la tâche, la phase de négociation avec l'enseignant mettra au point les modalités et la stratégie lui permettant de réaliser son 
objectif et définira les critères et les indices lui permettant d'évaluer la qualité des processus et du produit envisagé.

Après la réalisation de la tâche, l'apprenant la présente à son public. Cette phase de présentation sera suivie d'une évaluation par le groupe et d'une auto-évaluation. Il y aura donc des moments de réflexion permettant de faire le point sur les acquis et les processus ayant mené à ces acquis.

\section{Tableau 1 : Le cycle de la tâche}

\begin{tabular}{|c|l|l|}
\hline \multirow{2}{*}{$\begin{array}{c}\text { La pré- } \\
\text { tâche }\end{array}$} & orientation sur la tâche & $\begin{array}{l}\text { lien avec la vie réelle et } \\
\text { mobilisation des pré-connaissances }\end{array}$ \\
\cline { 2 - 4 } & négociation & $\begin{array}{l}\text { négociation de la tâche et des } \\
\text { modalités d'exécution : qualité } \\
\text { requise du produit fini, gestion du } \\
\text { temps et de l'espace }\end{array}$ \\
\hline \multirow{2}{*}{ La tâche } & ressources & matériaux requis, aide disponible \\
\hline & elanification & démarche et plan \\
\hline \multirow{2}{*}{$\begin{array}{c}\text { La post- } \\
\text { tâche }\end{array}$} & présentation de la tâche & exécution de la tâche \\
\cline { 2 - 3 } & réflexion et feed-back & $\begin{array}{l}\text { évaluation, autoévaluation } \\
\text { énumération des acquis et } \\
\text { évaluation de la démarche }\end{array}$ \\
\cline { 2 - 3 } & structuration des acquis & $\begin{array}{l}\text { cadres de référence, schémas... } \\
\text { replaçant les acquis dans le } \\
\text { contexte du système }\end{array}$ \\
\hline
\end{tabular}

\section{Conclusion}

En adoptant l'approche par les tâches, le CECR nous a apporté de nouvelles perspectives de conception et d'élaboration de cours. La mise en œuvre d'une tâche efficace renforce le processus d'apprentissage. Elle incite naturellement les apprenants à comprendre leurs propres processus d'apprentissage.

Comme on a pu le remarquer, l'approche par les tâches constitue une sorte de renouveau pédagogique pour les classes. L'introduction des tâches 
en classe peut s'avérer très utile pour les apprenants. Elle sert à activer l'apprentissage.

Par ailleurs, le recours aux tâches qui simulent des situations réelles préparent les apprenants à l'exécution des tâches réelles et donc à l'insertion dans la société où se passent les interactions.

\section{Les outils de la recherche et l'expérimentation}

Il est important pour le Ministère de l'enseignement supérieur et ses partenaires, de former les futurs-enseignants à la gestion responsable de la classe. Celle-ci constitue en effet l'atelier approprié où se réalise l'œuvre éducative.

La classe égyptienne respecte peu les normes pédagogiques indispensables pour un apprentissage efficace. La chercheuse élabore un module de formation pour remédier à cette situation.

Ce module de formation constitue un document utile de référence pour la gestion rationnelle d'une classe tant au niveau du primaire qu'au niveau secondaire.

\section{Le module de formation}

Ce module (annexe 1) contient 15 activités regroupées en 8 thèmes majeurs.

Chaque sujet d'activité est développé dans une fiche pédagogique à laquelle est jointe une fiche technique.

La fiche pédagogique de chaque activité contient les sept points suivants : le titre de l'activité, les objectifs spécifiques, la durée, le déroulement de l'activité et les questions de synthèse. 
Quant à la fiche technique, elle détaille et explicite des informations à communiquer au public cible.

Ce module vise, en général, à sensibiliser l'étudiant-maître sur l'importance de la classe dans l'œuvre éducative, à améliorer sa gestion dans son contexte actuel, à enrichir les méthodes et techniques d'enseignement, à renforcer les compétences de l'étudiant-maître.

\section{Les objectifs du Module de Formation}

\section{Objectif général}

Permettre à l'étudiant-maître d'acquérir et/ou de développer des connaissances, des attitudes et des comportements susceptibles d'améliorer la gestion d'une classe du FLE.

\section{Objectifs spécifiques}

Permettre à l'étudiant-maître de :

- Identifier, fabriquer et exploiter les supports pédagogiques

- Tenir et faire tenir correctement les documents pédagogiques

- Se servir correctement des méthodes d'organisation d'une classe

- Utiliser d'une manière appropriée les techniques d'organisation de classe

- Renforcer ses compétences sur les différents types d'évaluation

- Améliorer le climat de travail dans sa classe

- Organiser des activités scolaires et parascolaires

- Planifier une séquence didactique selon la pédagogique par objectif

\section{Les thèmes du Module de Formation}

- La constitution d'un groupe

- Les techniques d'organisation d'une classe

- Le climat de travail en classe 
- Les méthodes et les techniques d'enseignement

- Les supports pédagogiques

- Les documents pédagogiques

- L'évaluation pédagogique

- La pédagogie par objectifs

Les unités du Module de Formation

Unité 1

Thème 1 : La constitution d'un groupe

Activité 1 : Présentation des participants par l'Interview 2 à 2.

Activité 2 : Règles de fonctionnement

Thème 2 : Les techniques d'organisation d'une classe

Activité 1 : Composition d'une classe

Activité 2 : Techniques d'organisation d'une classe

Unité 2

Thème 3 : Le climat de travail en classe

Activité 1 : Attitudes et comportements des enseignants

Activité 2: Attitudes et comportements des élèves

Thème 4: Les méthodes et les techniques d'enseignement

Activité 1 : Méthodes et techniques actives et participatives

Activité 2 : Activités scolaires et parascolaires

\section{Unité 3}

Thème 5 : Les supports pédagogiques

Activité 1 : Fabrication et exploitation du matériel didactique

Activité 2 : Usage adéquat des moyens d'enseignement

Thème 6 : Les documents pédagogiques

Activité 1 : Tenue des documents pédagogiques 


\section{Unité 4}

Thème 7: L'évaluation pédagogique

Activité 1 : Types d'évaluation pédagogique

Activité 2 : Moyens d'évaluation

Thème 8 : La pédagogie par objectifs

Activité 1 : Formulation des objectifs pédagogiques

Activité 2 : Elaboration d'une fiche pédagogique

\section{Le pré-post test}

Le test (annexe 2) a pour objectifs de

- mesurer le degré d'acquisition des connaissances, des attitudes et des comportements susceptibles d'améliorer la gestion d'une classe du FLE.

- mesurer le degré d'efficacité du module de formation

Le pré-post test se compose de 10 exercices.

La note totale du test est 50 points.

\section{La grille d'observation}

Cette grille (annexe 3) sert à observer et ajuster la compétence de gestion de classe durant la passation des activités du module. Elle se compose de 12 items valorisant le savoir-agir des étudiantes.

\section{L'expérimentation}

Ce module de formation a été expérimenté sur une période de trois mois avec les étudiantes en $2^{\text {ème }}$ année à la faculté de Jeunes-Filles.

Les étapes de l'expérimentation

- La première séance est consacrée au test (pré-test) réalisé auprès de toutes les étudiantes (futurs-enseignantes).

- La chercheuse a exploité les unités didactiques composées de plusieurs activités, elle a utilisé la grille pour faire le suivi de quelques éléments. 
- Ensuite, elle a réappliqué le test (post-test) où elle évalue l'influence du module sur la gestion de classe.

- Enfin, elle analyse et interprète les résultats.

\section{Conclusion}

Le degré d'engagement des étudiantes dans les tâches dépend de la valeur qu'elles accordent à la tâche. Le rôle de l'enseignant consiste à favoriser l'implication maximale des étudiantes en leur permettant de jouer un rôle actif dans leurs apprentissages et en leur faisant voir la pertinence des activités. En proposant des activités stimulantes et riches de sens, l'enseignant incite les étudiantes à s'engager dans la tâche. Il s'assure par la suite de conserver l'attention et l'engagement des étudiantes tout au long de l'activité par diverses stratégies.

Une stratégie fréquemment utilisée pour conserver l'attention des étudiantes est de leur poser diverses questions pour qu'elles cherchent la réponse dans leur tête. En réfléchissant pour trouver une solution, elles restent actives et engagées jusqu'à la fin.

L'évaluation formative (la grille) et sommative (le test) permettent à l'enseignant de se renseigner sur les apprentissages, les progrès et les difficultés des étudiantes. Par la suite, il fait des retours sur la matière qui semble moins comprise afin de s'assurer que toutes développent les compétences visées par le module de formation.

\section{L'analyse et l'interprétation des résultats}

L'analyse porte sur les progrès réalisés entre le pré-post test, sur l'effet de l'utilisation du module sur la gestion de classe du FLE chez l'échantillon Pour l'analyse statistique des résultats, la chercheuse a calculé la moyenne arithmétique des notes, l'écart type et la valeur de"T". 


\section{Tableau 2 : les résultats obtenus au pré-post test}

de la compétence de gestion de classe

\begin{tabular}{|c|c|c|c|c|c|c|}
\hline Mesure & $\mathbf{n}$ & TN & TC & M & E & T \\
\cline { 1 - 6 } Pré-test & \multirow{2}{*}{24} & 416 & 9052 & 17,3 & 8,8 & \multirow{2}{*}{9,37} \\
\cline { 1 - 5 } Post-test & & 1022 & 45961 & 42,6 & 10,04 & \\
\hline
\end{tabular}

Le tableau ci-dessus indique le total des notes, le total des carrés de ces notes, la moyenne arithmétique, l'écart type et la valeur de " $\mathrm{T}$ " des étudiantes au pré-post test.

Notons que:

La différence entre la moyenne des notes des étudiantes au pré-post test est significative au niveau de 0.01 , en faveur du groupe après l'étude expérimentale. Ce qui valorise l'efficacité du module de formation proposé.

\section{Discussion}

Quelques éléments dégagés de l'observation des activités du module :

- des actions sont menées en vue d'organiser l'environnement au niveau spatial et au niveau matériel ainsi que des actions sont réalisées en vue de changer cet environnement ;

- des actions sont menées en relation à la dimension de l'organisation du groupe classe pour l'activité et des actions sont réalisées en vue de modifier cette organisation.

- des actions sont menées en relation à la dimension sociale de la classe et à l'aménagement du code de vie. Elles consistent en communications destinées à mettre à la disposition de toutes les étudiantes les informations nécessaires.

- des actions sont menées en relation à la dimension temporelle destinées à organiser les tâches au sein des moments didactiques en fonction du 
temps disponible et des actions de réorganisation de l'espace temps qui reste disponible.

- des actions sont réalisées en fonction des comportements des étudiantes, des actions de contrôle, des sollicitations auprès des étudiantes à ce qu'elles réalisent elles-mêmes des actions nouvelles, qu'elles adoptent des comportements plus appropriés lors de la réalisation des activités

- des actions de régulation à caractère interactif sont réalisées. Elles ont pour but d'engager une relation particulière avec les étudiantes et sollicitent celles-ci à réagir, à modifier des comportements ou des attitudes adoptées.

\section{Conclusion}

Pour conclure notre étude, il nous semble opportun de revenir à la question posée, qui ouvrait sur les actions de gestion de classe et la capacité des futurs enseignants à entreprendre de telles actions.

La formalisation de certaines actions et l'explication du caractère contraignant des situations vécues par les futurs enseignants sont des informations intéressantes mentionnées dans notre étude.

La méthodologie, utilisée dans cette recherche, met en relief la construction de cette compétence pendant la formation initiale, en permettant au futur enseignant d'analyser sa relation à une situation professionnelle qui s'est avérée pour lui problématique et dont il fait récit. Elle s'accompagne aussi d'un développement de la capacité à structurer la réflexion.

En ce sens, la finalité de l'exploitation du module proposé touche à un principe éthique, qui fonde la formation des futurs enseignants : aider à la construction d'une identité professionnelle progressivement assurée et construire la compétence de gérer sa classe, en vue de faciliter l'adaptation

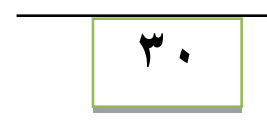


aux interactions en classe; aussi bien que donner l'occasion aux futurs enseignants de se livrer, avec l'aide du formateur, à une réflexion sur les difficultés rencontrées en matière de gestion de classe. Il s'agit là, d'une orientation dans le travail de formation qui prend toute sa place dans la formation initiale, parce qu'elle peut justement permettre l'ajustement des représentations des futurs enseignants par rapport aux pratiques à envisager dans les situations difficiles, en l'outillant progressivement de catégories descriptives et d'analyse.

\section{Bibliographie}

1. Archambault, J. et Chouinard., R. (2003). Vers une gestion éducative de la classe. 2e édition. Boucherville: Gaëtan Morin.

2. Arlin, M. (1979). Teacher transitions can disrupt time flow in classrooms. American Educational Research Journal, 16, A2-56.

3. Bérard, É. (2009).Les tâches dans l'enseignement du FLE : rapport à la réalité et dimension didactique. Le français dans le monde, $\mathrm{n}^{\circ}$ spécial «La perspective actionnelle et l'approche par les tâches en classe de langue », pp. 36-44.

4. Beyda, S. D., Zentall, S. S. et Ferko, D. J. K. (2002). The relationship between teacher practices and the task appropriate and social behavior of student with behavioural disorders. Behavioral Disorders. 27(3), 236255.

5. Bowen, F., Desbiens, N., Gendron, M. et Bélanger, J. (2005). L'acquisition et le développement des habiletés sociales. In. N. Desbiens, C. Lanaris et L. Massé. (dir), Les troubles du comportement à l'école : Prévention, évaluation et intervention. (p. 213-227). Québec : Gaétan Morin. 
6. Brophy, J.E.(1988). Education teachers about managing classrooms and students. Teaching and Teacher Education, 4, 1-18.

7. Bru, E., Stephens, P. et Torsheim, T. (2002). Students' perceptions of class management and reports of their own misbehavior. Journal of School Psychology. 40(4), 287- 307.

8. Burden, RR. (1995). Classroom management and discipline. Methods to facilitate cooperation and instruction. New York (NY) : Longman.

9. Charlier, E. (1989). Planifier un cours, c'est prendre des décisions. Bruxelles: De Boeck.

10.Chouinard, R. (1999). Enseignants débutants et pratiques de gestion de classe. Revue des sciences de l'éducation. 25(3), 497-514.

11.Chouinard, R., Plouffe, C. et Archambault, J. (2005). Le soutien de la motivation scolaire. In. N. Desbiens, C. Lanaris et L. Massé. (dir), Les troubles du comportement à l'école : Prévention, évaluation et intervention. (p. 261-270). Québec : Gaétan Morin.

12.Clark, CM. et Peterson, P.L. (1986). Teachers though processes. In M.C. Wittrock (dir.), Handbook of Research on Teaching (3e éd.) (p. 255-295). New York (NY) : Macmillan.

13.Conseil de l'Europe. (2001).Cadre européen commun de référence pour les langues, Paris, Didier.

14.Conseil de l'Europe. Guide pour les utilisateurs, Strasbourg. (2002) http://www.coe.int/t/dg4/portfolio/documents/guide-pour-utilisateursavril02.doc

15.Conseil supérieur de l'éducation. (1995). Pour une gestion de la classe plus dynamique au secondaire. 
16.Coste, D. (2009).Tâche, progression, curriculum. Le français dans le monde, $\mathrm{n}^{\circ}$ spécial «La perspective actionnelle et l'approche par les tâches en classe de langue », pp. 15-24.

17.DA COSTA, Paulo. (2002).Compétence de communication : Le pivot abstrait de la didactique des langues, Revista Kulonga (Revista das ciências da educação e estudos multidisciplinares), n², avril, p.43-60.

18.Desgagné, S. (1994). À propos de la «discipline de classe»:analyse du savoir professionnel d'enseignantes et enseignants expérimentés du secondaire en situation de parrainer des débutants. Thèse de doctorat. Québec : Université Laval.

19.Domalga-Zysk, E. (2006). The significance of adolescents' relationships with significant others and school failure. School Psychology International, 27(2), 232-247.

20.Doyle, W. (1986). Classroom organization and management. In M.C. Wittrock (dir.), Handbook of Research on Teaching (p. 392-431). New York (NY) : Macmillan.

21.Ellis, R. (2003).Task-based Language Learning and Teaching. Oxford : Oxford University Press.

22.Emmer, E. T. et Stough, L. M. (2001). Classroom management: A critical part of teacher educational psychology, with implications for teacher education. Educational Psychologist. 36(2), 103-112.

23.Fijalkow, J. (1996). Mauvais lecteurs, pourquoi? Paris : Presses universitaires de France.

24.Freinet, C. (1994). OEuvres pédagogiques. Paris: Seuil.

25.Hill, G. (1944). Teachers' instructional difficulties - A review of research. Journal of Educational Research, 37(8), 602-615. 
26.Johnson, D. D., Rice, M. P., Edgington, W. D. et Williams, P. (2005). How to succeed in classroom. Kappa Delta Pi Record. 42(1), 28-32.

27.Jones, EH. (1987). Positive classroom discipline. New York (NY) : McGraw-Hill.

28.Kazeroni, A. (2004).La construction d'une tâche d'apprentissage d'une langue étrangère dans des environnements informatiques, Études de Linguistique Appliquée, pp. 159-171.

29.Klem, A. M. et Connell, J. P. (2004). Relationships matter: Linking teacher support to student engagement and achievement. The Journal of School Health. 74(7), 262- 273.

30.Kounin, J.S. (1970). Discipline and group management in classrooms. New York (NY): Holt, Rinehart and Winston.

31.Lapointe, J. M. et Legault, F. (2004). Les relations avec l'entourage et l'adaptation psychosociale à l'école secondaire. Canadian Journal of Behavioural Science.36(3), 244-254.

32.Lessard, A. ; Schmidt, S. (2011). Recension des écrits sur la Gestion de Classe. Université de Sherbrooke

33.Le français dans le monde, $\mathrm{n}^{\circ}$ spécial (2009). «La perspective actionnelle et l'approche par les tâches en classe de langue». Clé International, Paris.

34.Leinhardt, G., Weidman, C. et Hammond, K.M. (1987). Introduction and Integration of Classroom Routines by Expert Teachers. Curriculum Inquiry, 17(2), 135-176.

35.Martineau, S., Gauthier, C. et Desbiens, J.-F. (1999). La gestion de classe au cœur de l'effet enseignant. Revue des sciences de l'éducation. 25(3), 467-496. 
36.McQueen,T (1992). Essentials of classroom management and discipline. New York (NY): Harper Collins.

37.Muller, C. (2001). The role of caring in the teacher-student relationship for at-risk students. Sociological Inquiry. 71(2), 241-255.

38.Murdock, T. B., and Miller, A. (2003). Teachers as sources of middle school students' motivational identity: variable-centered and personcentered analytic approaches. The Elementary School Journal. 103(4), 383-399.

39.Narcy-Combes, J-P., Walski, J. (2004).Le concept de tâche soumis au crible de nouvelles questions. Les Cahiers de l'APLIUT, vol XXIII, pp. 27-44.

40.Nault, T (1993).Etude exploratoire de l'insertion professionnelle des enseignants débutants au niveau secondaire. Thèse de doctorat. Montréal : Université de Montréal.

41.Nault, T (1998). L'enseignant et la gestion de classe. Montréal: Les Éditions Logiques.

42.Nault, T (1999). Eclosion d'un moi professionnel personnalisé et modalités de préparation aux premières rencontres d'une classe. In J.-C. Hétu, M. Lavoie et S. Baillauquès (dir.), Jeunes enseignants et insertion professionnelle (p. 139-161). Bruxelles: De Boeck Université.

43.Nault, T. (1997). La gestion de classe et ses spécificités. In Comité d'organisation de l'A.E.C.S.E. (dir.), Les actes du Deuxième congrès d'actualité de la recherche en éducation et formation (p. 307-310). France : Nanterre.

44.Nunan, D. (1989). Designing Tasks for the Communicative Classroom. Cambridge : Cambridge University Press. 
45.Overton, T. (2004). Promoting academic success through environmental assessment. Intervention in School and Clinic. 39(3), 147-153.

46.Pêcheur, J. (2010). Rôle des interactions dans l'approche actionnelle, présenté le 24/10/2010 au 7e Congrès Panhellénique et International des Professeurs de Français, Athènes.

47.Puren, C. (2007).Enseigner et apprendre les langues sur un mode qui change avec le monde: L'exemple du passage de l'approche communicative à la perspective actionnelle». Seminario Nazionale LEND Bologna 18-19-20

48.Puren, C. (2009).Conclusion-synthèse : variation sur la perspective de l'agir social en didactique des langues-cultures étrangères. Le français dans le monde, $\mathrm{n}^{\circ}$ spécial «La perspective actionnelle et l'approche par les tâches en classe de langue », pp. 154-168.

49.Puren, C. (2006).De l'approche communicative à la perspective actionnelle, Le français dans le monde, 347, 37-40.

50.Puren, C. (2006).La perspective actionnelle: vers une nouvelle cohérence didactique, Le français dans le monde, 348, 42-44.

51.Reynolds, A. (1995). The Knowledge Base for Beginning Teachers: Education Professionals' Expectations versus Research Findings on Learning to Teach. The Elementary School Journal. 95(3), 199-221.

52.Richardson, V. et Fallona, C. (2001). Classroom management as method and manner. Journal of Curriculum Studies. 33(6), 705-728.

53.Robert, J-P. (2008). Dictionnaire pratique de didactique du FLE. Ophrys, Paris. 
54.Rosen, É. (2009). La perspective actionnelle et l'approche par la tâche. Le français dans le monde, $\mathrm{n}^{\circ}$ spécial «La perspective actionnelle et l'approche par les tâches en classe de langue », pp. 6-14.

55.Rosenberg, M. S. et Jackman, L. A. (2003). Development, implementation, and sustainability of comprehensive school-wide behavior management systems. Intervention in School and Clinic. 39(1), $10-21$.

56.Ryan, A. M. et Patrick, H. (2001). The social environment and changes in adolescents'motivation and engagement during middle school. American Educational Research Journal. 38(2), 437-460.

57.Sigler, E. A. et Aamidor, S. (2005). From positive reinforcement to positive behaviors: An everyday guide for the practitioner. Early Childhood Educational Journal. 32(4), 249-253.

58.Smith, S. W. et Gilles, D. L. (2003). Using key instructional elements to systematically promote social skill generalization for students with challenging behavior. Intervention in School and Clinic. 39(1), 30-37.

59.Springer, C. (2009). La dimension sociale dans le CECR : pistes pour scénariser, évaluer et valoriser l'apprentissage collaboratif. Le français dans le monde, $\mathrm{n}^{\circ}$ spécial «La perspective actionnelle et l'approche par les tâches en classe de langue », pp. 25-35.

60.Struyk, L.R. (1991). Self-Evaluation Procedures for Teachers. Document ERIC ED336361.

61.Willis, J. (1996). A Framework for Task-Based Learning. Harlow: Longman.

62.Wolfgang, CH. (1999). Solving discipline problems. Boston: Allyn and Bacon. 
63. Yinger, R. (1979). Routines in teacher planning. Theory Into Practice, XVIII(3), 163-169.

64.Yorke, D.B. (1998). Norm setting: Rules by and for the students. Vocational Education Journal, 63(5), 32, 33 et 47. 